PROCEEDINGS OF THE

AMERICAN MATHEMATICAL SOCIETY

Volume 126, Number 12, December 1998, Pages 3561-3569

S $0002-9939(98) 04450-5$

\title{
ZEROS OF THE ZAK TRANSFORM ON LOCALLY COMPACT ABELIAN GROUPS
}

\author{
EBERHARD KANIUTH AND GITTA KUTYNIOK
}

(Communicated by J. Marshall Ash)

\begin{abstract}
Let $G$ be a locally compact abelian group. The notion of Zak transform on $L^{2}\left(\mathbb{R}^{d}\right)$ extends to $L^{2}(G)$. Suppose that $G$ is compactly generated and its connected component of the identity is non-compact. Generalizing a classical result for $L^{2}(\mathbb{R})$, we then prove that if $f \in L^{2}(G)$ is such that its Zak transform $Z f$ is continuous on $G \times \widehat{G}$, then $Z f$ has a zero.
\end{abstract}

\section{INTRODUCTION}

The Zak transform on the real line, sometimes also referred to as the WeilBrezin map, was introduced in 1967 by Zak [11] to construct a quantum mechanical representation for the description of the motion of a Bloch electron in the presence of a magnetic or electric field. Subsequently it proved to be an important tool in applied areas such as signal theory, wavelet analysis and solid state physics (compare the survey article [7] and the references therein).

For $f \in L^{2}(\mathbb{R})$ the Zak transform $Z f$ is the function on $\mathbb{R} \times \mathbb{R}$ defined by

$$
Z f(x, y)=\sum_{k=-\infty}^{\infty} f(x+k) e^{2 \pi i y k} .
$$

A striking property of the Zak transform, independently shown by Zak [3] and Janssen [6], is that $Z f$ has a zero whenever $Z f$ is continuous on $\mathbb{R} \times \mathbb{R}$. Actually, in certain special cases like when $f$ is the Gaussian, this follows from elementary properties of theta series.

Now, the notion of the Zak transform admits a natural generalization to locally compact abelian groups (see Section 3). Given a locally compact abelian group $G$, its dual group $\widehat{G}$ and a uniform lattice $K$ in $G$, the Zak transform, associated to $K$, of $f \in L^{2}(G)$ can be defined (almost everywhere) on $G \times \widehat{G}$ by

$$
Z f(x, \omega)=\sum_{k \in K} f(x k) \omega(k) .
$$

The main purpose of this note is to extend the above result to compactly generated locally compact abelian groups. In fact, we are going to establish the following stronger result.

Received by the editors October 1, 1996 and, in revised form, April 20, 1997.

1991 Mathematics Subject Classification. Primary 43A32; Secondary 43A15, 43A40.

(C)1998 American Mathematical Society 
Theorem. Let $G$ be a compactly generated locally compact abelian group with noncompact connected component of the identity, $K$ a uniform lattice in $G$ and $\Gamma$ the annihilator of $K$ in the dual group $\widehat{G}$ of $G$. Suppose that $g: G \times \widehat{G} \rightarrow \mathbb{C}$ is a continuous function satisfying the quasi-periodicity relation

$$
g(x k, \omega \gamma)=\overline{\omega(k)} g(x, \omega)
$$

for all $(x, \omega) \in G \times \widehat{G}$ and $(k, \gamma) \in K \times \Gamma$. Then $g$ has a zero.

The converse to the theorem also holds (Remark 1 in Section 4). As an immediate consequence of the theorem we obtain the following:

Corollary. Let $G$ and $K$ be as in the theorem and $Z$ the associated Zak transform. Let $f \in L^{2}(G)$ and suppose that $Z f$ is continuous on $G \times \widehat{G}$. Then $Z f$ has a zero.

It is worthwhile to point out that conversely the corollary implies the theorem at least when $G$ is first countable (compare Remark 5).

The proof of the theorem will be given in Section 2, whereas in Section 3 we deal with the Zak transform. Finally, in Section 4 we conclude with some remarks.

\section{Proof of the theorem}

Let $G$ be an arbitrary locally compact abelian group and let $K$ be a uniform lattice in $G$, that is, a discrete subgroup of $G$ with compact quotient group $G / K$. In the sequel, $\Gamma$ will denote the annihilator of $K$ in $\widehat{G}$,

$$
\Gamma=A(K, \widehat{G})=\{\gamma \in \widehat{G}: \gamma(k)=1 \text { for all } k \in K\} .
$$

Then $\Gamma$ is a uniform lattice in $\widehat{G}$ since $\Gamma$ is topologically isomorphic to $\widehat{G / K}$ and $\widehat{G} / \Gamma$ is topologically isomorphic to $\widehat{K}$ (via the restriction map $\omega \Gamma \rightarrow \omega \mid K$ ). The following lemma is required in the proof of the theorem.

Lemma 1. Let $\mathcal{H}$ be a downward directed system of compact subgroups of $G$ (with normalized Haar measures) such that $\bigcap_{H \in \mathcal{H}} H=\{e\}$. Let $g$ be a continuous function on $G \times \widehat{G}$ such that

$$
g(x k, \omega \gamma)=\overline{\omega(k)} g(x, \omega)
$$

for all $(x, \omega) \in G \times \widehat{G}$ and $(k, \gamma) \in K \times \Gamma$. For each $H \in \mathcal{H}$, define $g_{H}$ on $G \times \widehat{G}$ by

$$
g_{H}(x, \omega)=\int_{H} g(x h, \omega) d h .
$$

Then $g_{H}$ is continuous and satisfies $g_{H}(x k, \omega \gamma)=\overline{\omega(k)} g_{H}(x, \omega)$. If every $g_{H}$ has a zero, then $g$ has a zero.

Proof. That $g_{H}$ is continuous follows immediately from the uniform continuity of $g$ on compact subsets of $G \times \widehat{G}$. Moreover, for $(x, \omega) \in G \times \widehat{G}$ and $(k, \gamma) \in K \times \Gamma$,

$$
g_{H}(x k, \omega \gamma)=\int_{H} g(x k h, \omega) d h=\overline{\omega(k)} \int_{H} g(x h, \omega) d h=\overline{\omega(k)} g_{H}(x, \omega) .
$$

Now suppose that every $g_{H}$ has a zero. Since $G / K$ and $\widehat{G} / \Gamma$ are compact, there exist compact subsets $C$ of $G$ and $\Delta$ of $\widehat{G}$ such that $G=C K$ and $\widehat{G}=\Delta \Gamma$. Due to the quasi-periodicity, for each $H \in \mathcal{H}$ there exist $x_{H} \in C$ and $\omega_{H} \in \Delta$ such that $g_{H}\left(x_{H}, \omega_{H}\right)=0$. $C$ and $\Delta$ being compact, by passing to a subnet if necessary, 
we can assume that $x_{H} \rightarrow x$ and $\omega_{H} \rightarrow \omega$ for some $x \in C$ and $\omega \in \Delta$. Finally, employing the uniform continuity of $g$ on compact sets once more, we obtain that

$$
\begin{gathered}
|g(x, \omega)|=\left|g(x, \omega)-g_{H}\left(x_{H}, \omega_{H}\right)\right| \\
=\left|\int_{H}\left(g(x, \omega)-g\left(x_{H} h, \omega_{H}\right)\right) d h\right| \leq \int_{H}\left|g(x, \omega)-g\left(x_{H} h, \omega_{H}\right)\right| d h,
\end{gathered}
$$

which converges to zero as $H \rightarrow\{e\}$.

We now turn to the proof of the theorem. Notice first that by the structure theorem for compactly generated locally compact abelian groups [5, Theorem 9.8], $G$ is of the form $G=\mathbb{R}^{p} \times \mathbb{Z}^{q} \times C$ where $C$ is a compact group and $p \geq 1$ since by hypothesis $G$ has a non-compact connected component of the identity. Now, compact groups are projective limits of Lie groups [10, p.99]. Therefore, there exists a system $\mathcal{H}$ of closed subgroups $H$ of $C$ as in Lemma 1 such that $C / H$ is a Lie group for every $H \in \mathcal{H}$. Thus, for each $H \in \mathcal{H}$, there is a closed subgroup $L_{H}$ of $C$ such that $H \subseteq L_{H}, L_{H}$ is of finite index in $C$ and $L_{H} / H=\mathbb{T}^{r_{H}}$ for some $r_{H} \in \mathbb{N}_{0}$.

By Lemma 1, for any such $H, g_{H}$ is continuous, and once we have established that $g_{H}$ has a zero on $G \times \widehat{G}$, it follows that $g$ has a zero as well. To that end, fix $H$ and set $L=L_{H}$ and $r=r_{H}$. Replacing $g_{H}$ by $g$, we can therefore assume that $g$ is constant on cosets of $H$. Let $\pi: G \rightarrow G / H$ denote the quotient homomorphism. Then $\pi(K)=K H / H$ is a uniform lattice in $G / H$, and

$$
A(\pi(K), \widehat{G / H})=\{\chi \in \widehat{G / H}: \chi \circ \pi \in A(K, \widehat{G})\} .
$$

Now, the function $\tilde{g}: G / H \times \widehat{G / H} \rightarrow \mathbb{C}$ defined by

$$
\tilde{g}(\pi(x), \chi)=g(x, \chi \circ \pi),
$$

$x \in G, \chi \in \widehat{G / H}$, is continuous and satisfies the equation

$$
\tilde{g}(\pi(x) \pi(k), \chi \delta)=\tilde{g}(\pi(x), \chi) \overline{(\chi \circ \pi)(k)}
$$

for all $x \in G, k \in K, \chi \in \widehat{G / H}$ and $\delta \in A(\pi(K), \widehat{G / H})$. It suffices to show that $\tilde{g}$ has a zero. Thus, after moving to $G / H$, we can assume that $L=\mathbb{T}^{r}$. Towards a contradiction, suppose that $g(x, \omega) \neq 0$ for all $(x, \omega) \in G \times \widehat{G}$.

In what follows, for $x \in G$ and $\omega \in \widehat{G}$, let $x_{1} \in \mathbb{R}^{p}$ and $\omega_{1} \in \widehat{\mathbb{R}^{p}}$ denote the first component of $x$ and $\omega$, respectively. When convenient, we shall identify $\mathbb{R}^{p}$ with $\widehat{\mathbb{R}^{p}}$ by writing $\omega_{1}\left(x_{1}\right)=\exp 2 \pi i\left\langle x_{1}, \omega_{1}\right\rangle$. Let $1_{C}$ be the trivial character of $C$ and $e_{r}: \mathbb{R}^{r} \rightarrow \mathbb{T}^{r}$ the covering homomorphism given by

$$
e_{r}(u)=\left(e^{2 \pi i u_{1}}, \ldots, e^{2 \pi i u_{r}}\right)
$$

for $u=\left(u_{1}, \ldots, u_{r}\right) \in \mathbb{R}^{r}$. We define homomorphisms

$$
\varphi_{1}: \mathbb{R}^{p} \times \mathbb{R}^{r} \rightarrow \mathbb{R}^{p} \times\{0\} \times \mathbb{T}^{r} \subseteq G, \quad\left(x_{1}, u\right) \rightarrow\left(x_{1}, 0, e_{r}(u)\right)
$$

and

$$
\varphi_{2}: \widehat{\mathbb{R}^{p}} \times \widehat{\mathbb{R}^{q}} \rightarrow \widehat{\mathbb{R}^{p}} \times \widehat{\mathbb{Z}^{q}} \times\left\{1_{C}\right\} \subseteq \widehat{G}, \quad\left(\omega_{1}, \chi\right) \rightarrow\left(\omega_{1},\left.\chi\right|_{\mathbb{Z}^{q}}, 1_{C}\right) .
$$

Since $g$ is continuous and has no zero on $G \times \widehat{G}$, we can consider the continuous function

$$
\left(x_{1}, u, \omega_{1}, \chi\right) \rightarrow \frac{g\left(\varphi_{1}\left(x_{1}, u\right), \varphi_{2}\left(\omega_{1}, \chi\right)\right)}{\left|g\left(\varphi_{1}\left(x_{1}, u\right), \varphi_{2}\left(\omega_{1}, \chi\right)\right)\right|}
$$


on $S=\mathbb{R}^{p} \times \mathbb{R}^{r} \times \widehat{\mathbb{R}^{p}} \times \widehat{\mathbb{R}^{q}}$. Since $S$ is simply connected, there exists a continuous function $\varphi: S \rightarrow \mathbb{R}$ such that

$$
\exp 2 \pi i \varphi\left(x^{\prime}, \omega^{\prime}\right)=\frac{g\left(\varphi_{1}\left(x^{\prime}\right), \varphi_{2}\left(\omega^{\prime}\right)\right)}{\left|g\left(\varphi_{1}\left(x^{\prime}\right), \varphi_{2}\left(\omega^{\prime}\right)\right)\right|}
$$

for all $x^{\prime} \in \mathbb{R}^{p} \times \mathbb{R}^{r}$ and $\omega^{\prime} \in \widehat{\mathbb{R}^{p}} \times \widehat{\mathbb{R}^{q}}$. Since $\varphi_{1}$ and $\varphi_{2}$ are homomorphisms, the quasi-periodicity relation for $g$ implies that

$$
\begin{gathered}
\exp 2 \pi i\left[\varphi\left(x^{\prime} k^{\prime}, \omega^{\prime}\right)-\varphi\left(x^{\prime}, \omega^{\prime}\right)\right]=\overline{\varphi_{2}\left(\omega^{\prime}\right)\left(\varphi_{1}\left(k^{\prime}\right)\right)} \\
=\overline{\omega_{1}\left(k_{1}\right)}=\exp \left(-2 \pi i\left\langle\omega_{1}, k_{1}\right\rangle\right)
\end{gathered}
$$

for all $\left(x^{\prime}, \omega^{\prime}\right) \in S$ and $k^{\prime} \in \varphi_{1}^{-1}(K)$, and

$$
\exp 2 \pi i\left[\varphi\left(x^{\prime}, \omega^{\prime} \gamma^{\prime}\right)-\varphi\left(x^{\prime}, \omega^{\prime}\right)\right]=1
$$

for all $\left(x^{\prime}, \omega^{\prime}\right) \in S$ and $\gamma^{\prime} \in \varphi_{2}^{-1}(\Gamma)$.

Since $S$ is connected and $\varphi$ is continuous, it follows that given $k^{\prime}$ and $\gamma^{\prime}$, there are integers $m_{1}\left(k^{\prime}\right)$ and $m_{2}\left(\gamma^{\prime}\right)$ such that

and

(1) $\varphi\left(x^{\prime} k^{\prime}, \omega^{\prime}\right)-\varphi\left(x^{\prime}, \omega^{\prime}\right)+\left\langle k_{1}, \omega_{1}\right\rangle=m_{1}\left(k^{\prime}\right)$

(2) $\varphi\left(x^{\prime}, \omega^{\prime} \gamma^{\prime}\right)-\varphi\left(x^{\prime}, \omega^{\prime}\right)=m_{2}\left(\gamma^{\prime}\right)$

for all $x^{\prime} \in \mathbb{R}^{p} \times \mathbb{R}^{r}$ and $\omega^{\prime} \in \widehat{\mathbb{R}^{p}} \times \widehat{\mathbb{R}^{q}}$. Applying (1) first and then (2) yields

$$
\begin{aligned}
\varphi\left(x^{\prime} k^{\prime}, \omega^{\prime} \gamma^{\prime}\right) & =\varphi\left(x^{\prime}, \omega^{\prime} \gamma^{\prime}\right)-\left\langle k_{1}, \omega_{1}\right\rangle-\left\langle k_{1}, \gamma_{1}\right\rangle+m_{1}\left(k^{\prime}\right) \\
& =\varphi\left(x^{\prime}, \omega^{\prime}\right)+m_{2}\left(\gamma^{\prime}\right)-\left\langle k_{1}, \omega_{1}\right\rangle-\left\langle k_{1}, \gamma_{1}\right\rangle+m_{1}\left(k^{\prime}\right) .
\end{aligned}
$$

On the other hand, applying (1) and (2) in the reverse order gives

$$
\begin{aligned}
\varphi\left(x^{\prime} k^{\prime}, \omega^{\prime} \gamma^{\prime}\right) & =\varphi\left(x^{\prime} k^{\prime}, \omega^{\prime}\right)+m_{2}\left(\gamma^{\prime}\right) \\
& =\varphi\left(x^{\prime}, \omega^{\prime}\right)-\left\langle k_{1}, \omega_{1}\right\rangle+m_{1}\left(k^{\prime}\right)+m_{2}\left(\gamma^{\prime}\right) .
\end{aligned}
$$

Subtracting these two equations shows that

$$
\left\langle k_{1}, \gamma_{1}\right\rangle=0
$$

for all pairs $\left(k_{1}, \gamma_{1}\right)$ such that $\left(k_{1}, 0, k_{3}\right) \in K$ for some $k_{3} \in \mathbb{T}^{r}$ and $\left(\gamma_{1}, \gamma_{2}, 1_{C}\right) \in \Gamma$ for some $\gamma_{2} \in \widehat{\mathbb{Z}^{q}}$.

We are now going to show that this is impossible. Notice first that, since $G^{\prime}=$ $\mathbb{R}^{p} \times L$ is open in $G, G^{\prime} /\left(G^{\prime} \cap K\right)$ is topologically isomorphic to $G^{\prime} K / K \subseteq G / K$, which is compact. Hence $K \cap G^{\prime}$ is cocompact in $G^{\prime}$. Let $K_{1}$ denote the set of first components of elements in $K \cap G^{\prime}$. Then $K_{1}$ contains a vector space basis for $\mathbb{R}^{p}$. Indeed, otherwise

$$
K \cap G^{\prime} \subseteq K_{1} \times L \subseteq V \times L \subseteq \mathbb{R}^{p} \times L=G^{\prime}
$$

for some proper subspace $V$ of $\mathbb{R}^{p}$, which contradicts the fact that $G^{\prime} /\left(K \cap G^{\prime}\right)$ is compact.

Thus it only remains to verify that there exist $\gamma_{1} \in \widehat{\mathbb{R}^{p}}$ and $\gamma_{2} \in \widehat{\mathbb{Z}^{q}}$ such that $\gamma_{1} \neq 0$ and $\left(\gamma_{1}, \gamma_{2}, 1_{C}\right) \in \Gamma$. Assume that $\left(\gamma_{1}, \gamma_{2}, 1_{C}\right) \in A(K, \widehat{G})$ only if $\gamma_{1}=0$. Then

$$
A(K C, \widehat{G}) \subseteq A\left(\mathbb{R}^{p} \times C, \widehat{G}\right),
$$

and hence $K C \supseteq \mathbb{R}^{p} \times C$, whence

$$
K /(K \cap C)=K C / C \supseteq \mathbb{R}^{p},
$$

which is impossible since $K$ is discrete. This finishes the proof of the theorem. 
The idea of writing $g /|g|$, when possible, as the exponential of some continuous function occurs already in the proofs that Zak [3] and Janssen [6] gave for the existence of a zero in the case $G=\mathbb{R}$. For a different proof compare [1, p.18].

\section{THE ZAK TRANSFORM AND ZEROS}

If $G$ is a locally compact abelian group and $K$ a uniform lattice in $G$, then a fundamental domain for $K$ will mean a Borel subset $S$ of $G$ such that every $x \in G$ can be uniquely written in the form $x=s k$ where $s \in S$ and $k \in K$.

Generalizing the classical notion of the Zak transform for the uniform lattice $\mathbb{Z}^{d}$ in $\mathbb{R}^{d}$, we are going to introduce the Zak transform on $L^{2}(G)$ associated to $K$. The first step is to guarantee the existence of a fundamental domain for $K$.

Lemma 2. Let $G$ be a locally compact abelian group and $K$ a uniform lattice in $G$. Then there exists a relatively compact fundamental domain for $K$.

Proof. We assume first that $G$ is compactly generated. Since $G$ is a projective limit of second countable groups [10, p.104] and $K$ is discrete, there exists a compact subgroup $C$ of $G$ such that $C \cap K=\{e\}$ and $G / C$ is second countable. By [8, Lemma 1.1] there exists a relatively compact fundamental domain $Q$ for $K C / C$ in $G / C$. Let $q: G \rightarrow G / C$ denote the quotient homomorphism, and set $S=q^{-1}(Q)$. Clearly, $S$ is a relatively compact Borel set, and using the fact that $K \cap C=\{e\}$, it is easy to check that $S$ is indeed a fundamental domain for $K$.

Now, drop the assumption that $G$ is compactly generated and choose an open compactly generated subgroup $H$ of $G$. Since $K \cap H$ is a uniform lattice in $H$, by the preceding paragraph there exists a relatively compact fundamental domain $S$ for $K \cap H$ in $H$. As $H$ is open and $G / K$ is compact, $K H$ has finite index in $G$. Let $F$ be a coset representative system for $K H$ in $G$, and let $T=F S$. Then $T$ is a relatively compact Borel set, and as above it is straightforward to verify that $T$ is a fundamental domain for $K$ in $G$.

By Lemma 2 there exist relatively compact fundamental domains $S$ for $K$ in $G$ and $\Omega$ for $\Gamma$ in $\widehat{G}$.

Let the Haar measure on $G$ be normalized so that Weil's formula holds, if we take on $G / K$ the normalized Haar measure and the counting measure on $K$. Clearly, if $G$ is $\sigma$-compact (equivalently, $K$ is countable), then $S$ has positive measure $(|S|>0)$. However, this is also true in the general case. To see this, choose a compactly generated open subgroup $H$ of $G$ containing $S$ and observe that $S k \cap H \neq \emptyset$ if and only if $k \in H$. Since $H$ is $\sigma$-compact and $K$ is discrete, there are only countably many such $k$. Thus $H$ is a countable union of sets $S k, k \in K$, whence $|S|>0$.

The map $\Phi: S \rightarrow G / K, x \rightarrow x K$ is a continuous bijection. For each measurable subset $M$ of $S$ and with $\chi_{M}$ the characteristic function of $M$, Weil's formula gives

$$
|M|=\int_{G} \chi_{M}(x) d x=\int_{G / K}\left(\sum_{k \in K} \chi_{M}(x k)\right) d(x K)=|\Phi M| .
$$

Hence $\Phi$ maps the measure on $S$ induced by the Haar measure on $G$ to the normalized Haar measure on $G / K$.

Similarly, normalizing the Haar measures on $\widehat{G}$ and $\widehat{G} / \Gamma$ appropriately, the mapping $\Omega \rightarrow \widehat{G} / \Gamma, \omega \rightarrow \omega \Gamma$ transforms the induced measure on $\Omega$ into the Haar measure on $\widehat{G} / \Gamma$, and $|\Omega|=1$. 
The next lemma will show that for an arbitrary locally compact abelian group the Zak transform can be defined as indicated in the introduction.

Lemma 3. Retain the preceding assumptions and notations, and let $f \in L^{2}(G)$. Then, for almost all $(x, \omega) \in S \times \Omega$,

$$
Z f(x, \omega)=\sum_{k \in K} f(x k) \omega(k)
$$

converges, and the function $Z f$ belongs to $L^{2}(S \times \Omega)$ and satisfies $\|Z f\|_{2}=\|f\|_{2}$.

Proof. For $k \in K$, define $f_{k} \in L^{2}(S \times \Omega)$ by $f_{k}(x, \omega)=f(x k) \omega(k)$. Then

$$
\sum_{k \in K}\left\|f_{k}\right\|_{2}^{2}=\sum_{k \in K} \int_{S} \int_{\Omega}\left|f_{k}(x, \omega)\right|^{2} d \omega d x=\sum_{k \in K} \int_{S}|f(x k)|^{2} d x=\|f\|_{2}^{2} .
$$

We claim that $\left\langle f_{k}, f_{l}\right\rangle=0$ for $k, l \in K, k \neq l$. To show this recall that if $C$ is a compact abelian group and $\varphi$ a non-trivial character of $C$, then $\int_{C} \varphi(y) d y=0[5$, Lemma 23.19]. Applying this to $C=\widehat{G} / \Gamma$ and the character $\varphi$ defined by

$$
\varphi(\omega \Gamma)=\omega\left(k l^{-1}\right), \quad \omega \in \widehat{G},
$$

we obtain

$$
\int_{\Omega} \omega\left(k l^{-1}\right) d \omega=\int_{\widehat{G} / \Gamma} \varphi(\omega \Gamma) d(\omega \Gamma)=0,
$$

and this in turn implies

$$
\left\langle f_{k}, f_{l}\right\rangle=\int_{S} \int_{\Omega} f(x k) \overline{f(x l)} \omega\left(k l^{-1}\right) d \omega d x=0 .
$$

It follows that the series $\sum_{k \in K} f_{k}$ converges in $L^{2}(S \times \Omega)$ and satisfies

$$
\left\|\sum_{k \in K} f_{k}\right\|_{2}^{2}=\sum_{k \in K}\left\|f_{k}\right\|_{2}^{2}=\|f\|_{2}^{2}
$$

In particular, $Z f(x, \omega)$ exists for almost all $(x, \omega) \in S \times \Omega$.

We can now define the Zak transform $Z f$ for $f \in L^{2}(G)$. Notice first that for every $(k, \gamma) \in K \times \Gamma$ and any finite subset $H$ of $K$,

$$
\sum_{h \in H} f(x k h)(\omega \gamma)(h)=\overline{\omega(k)} \sum_{l \in H} f(x l) \omega(l) .
$$

Thus $Z f(x k, \omega \gamma)$ converges if and only if $Z f(x, \omega)$ does. It follows from Lemma 3 that

$$
Z f(x, \omega)=\sum_{k \in K} f(x k) \omega(k)
$$

is defined for locally almost all $(x, \omega) \in G \times \widehat{G}$ (and, in fact, for a.a. $(x, \omega)$ if $G$ is $\sigma$-compact), and this function is called the Zak transform of $f$. We say that $Z f$ is continuous on $G \times \widehat{G}$ if there exists a continuous function $g$ on $G \times \widehat{G}$ which agrees with $Z f$ locally a.e. on $G \times \widehat{G}$. Of course, such a function $g$ then satisfies the quasi-periodicity relation $g(x k, \omega \gamma)=\overline{\omega(k)} g(x, \omega)$ for all $(x, \omega) \in G \times \widehat{G}$ and $(k, \gamma) \in K \times \Gamma$. Hence an application of the theorem yields the corollary. 


\section{SOME REMARKS}

We finish the paper by adding remarks concerning the hypotheses of the theorem and some application.

Remark 1. The converse to the theorem also holds. That is, if $G$ is a compactly generated locally compact abelian group, then $G_{0}$, the connected component of the identity, must be non-compact provided that $G$ has the following property: For every uniform lattice $K$ in $G$ and $f \in L^{2}(G), Z f$ has a zero whenever $Z f$ is continuous.

In fact, suppose that $G_{0}$ is compact so that $G=D \times C$ where $D$ is discrete and $C$ is compact. Choosing $K=D$ and $f=\chi_{C}$, one obtains for $x=d c, d \in D, c \in C$, and $\omega \in \widehat{G}$,

$$
Z f(x, \omega)=\sum_{k \in D} f(x k) \omega(k)=\overline{\omega(d)} .
$$

This formula shows that $Z f$ is continuous and of modulus 1 .

Remark 2 . In general, a locally compact abelian group $G$ need not contain a uniform lattice. The following example was kindly communicated by the referee.

Suppose $G$ is the group $\left(\mathbb{Z}_{4}\right)^{\infty}$ with the topology obtained when the subgroup $C$ generated by all elements of order 2 is declared to be open and compact. Then every discrete subgroup $K$ of $G$ has to be finite. Indeed, $K \cap C$ is finite and $x \rightarrow x^{2}$ is a homomorphism from $K$ into $K \cap C$ with kernel $K \cap C$.

However, if $G$ is of the form $G=\mathbb{R}^{p} \times D \times C$, where $D$ is discrete and $C$ is compact, then we can take $K=\mathbb{Z}^{p} \times D$. More specifically, if $G$ is compactly generated, say $G=\mathbb{R}^{p} \times \mathbb{Z}^{q} \times C$, then an abundance of uniform lattices can be constructed as follows. Let $h_{1}$ be a homomorphism of $\mathbb{Z}^{p} \subseteq \mathbb{R}^{p}$ into $C$ and let $h_{2}$ and $h_{3}$ be homomorphisms of $\mathbb{Z}^{q}$ into $\mathbb{R}^{p}$ and $C$, respectively. Then

$$
K=\left\{\left(x_{1}+h_{2}\left(x_{2}\right), x_{2}, h_{1}\left(x_{1}\right)+h_{3}\left(x_{2}\right)\right): x_{1} \in \mathbb{Z}^{p}, x_{2} \in \mathbb{Z}^{q}\right\}
$$

is a uniform lattice in $G$.

Remark 3. The condition that $Z f$ be continuous is satisfied whenever $f$ is continuous and rapidly decreasing outside of compact subsets of $G$. More precisely, it is well-known that if $f$ is a continuous function on $\mathbb{R}^{d}$ such that $|f(x)| \leq c\left(1+\|x\|_{2}\right)^{-\alpha}$ for some $\alpha>1$ and $c>0$, then $Z f$ is continuous. Slightly more general, it is not difficult to see that for $G=\mathbb{R}^{p} \times \mathbb{Z}^{q} \times C \subseteq \mathbb{R}^{p} \times \mathbb{R}^{q} \times C$, a similar hypothesis with respect to the $\mathbb{R}^{p}$ and $\mathbb{R}^{q}$ variables is sufficient.

For the two final remarks, let $K$ denote a uniform lattice in the locally compact abelian group $G, \Gamma$ the annihilator of $K$ in $\widehat{G}$ and $Z$ the Zak transform associated to $K$.

Remark 4. Let $S$ and $\Omega$ be relatively compact fundamental domains for $K$ in $G$ and $\Gamma$ in $\widehat{G}$, respectively. We have seen (Lemma 3 ) that, after suitably normalizing Haar measures, $Z$ maps $L^{2}(G)$ unitarily into $L^{2}(S \times \Omega)$. It can be shown that $Z$ is surjective provided that the mappings $S \rightarrow G / K$ and $\Omega \rightarrow \widehat{G} / \Gamma$ induce Hilbert space isomorphisms $L^{2}(S) \rightarrow L^{2}(G / K)$ and $L^{2}(\Omega) \rightarrow L^{2}(\widehat{G} / \Gamma)$, (compare the proof for $G=\mathbb{R}^{d}$ in [2]). This latter condition is satisfied if $S \rightarrow G / K$ and $\Omega \rightarrow \widehat{G} / \Gamma$ are Borel isomorphisms, that is, if $S$ and $\Omega$ arise from Borel cross-sections $G / K \rightarrow G$ 
and $\widehat{G} / \Gamma \rightarrow \widehat{G}$. Now, the existence of such cross-sections is guaranteed when $G$ (and hence $\widehat{G}$ ) is second countable ([8, Lemma 1.1] and [9, Theorem 4.2]).

Remark 5. Let $G$ be a first countable compactly generated locally compact abelian group, and let $K$ be a uniform lattice in $G$ and $\Gamma$ the annihilator of $K$ in $\widehat{G}$. Choose relatively compact Borel sets $S$ in $G$ and $\Omega$ in $\widehat{G}$ such that the quotient mappings are Borel isomorphisms (see Remark 4). Suppose that $g$ is a continuous function on $G \times \widehat{G}$ satisfying the quasi-periodicity relation $g(x k, \omega \gamma)=\overline{\omega(k)} g(x, \omega)$ for all $(x, \omega) \in G \times \widehat{G}$ and $(k, \gamma) \in K \times \Gamma$. Then, since $Z: L^{2}(G) \rightarrow L^{2}(S \times \Omega)$ is surjective, there exists $f \in L^{2}(G)$ such that $Z f=g$ a.e. on $S \times \Omega$, hence a.e. on $G \times \widehat{G}$. Thus, in this situation, the theorem and the corollary are equivalent.

Remark 6. Let $f \in L^{2}(G)$, and for $k \in K$ and $\gamma \in \Gamma$ define $\varphi_{k, \gamma} \in L^{2}(G)$ by $\varphi_{k, \gamma}(x)=\gamma(x) f\left(k^{-1} x\right)$. The collection of all these functions is called the Gabor system associated with $f$. In the classical situation, $G=\mathbb{R}^{d}$, the question of when this Gabor system forms a frame (an exact frame, an orthonormal basis) for $L^{2}(G)$ has been a matter of great interest.

In this context the Zak transform plays an important role. For instance, the set $\left\{\varphi_{k, \gamma}: k \in K, \gamma \in \Gamma\right\}$ constitutes a frame for $L^{2}\left(\mathbb{R}^{d}\right)$ with frame bounds $A$ and $B$ precisely when $A \leq|Z f| \leq B$ almost everywhere on $\mathbb{R}^{d} \times \widehat{\mathbb{R}^{d}}$ (see $[2$, Theorem 3.16]). Now, the proofs of these results carry over, in a straightforward manner, to a general locally compact abelian group $G$ provided that the mapping $Z: L^{2}(G) \rightarrow L^{2}(S \times \Omega)$ is onto. By the preceding remark we know this to be true for suitable $S$ and $\Omega$ at least when $G$ is second countable.

In particular, from the corollary we can draw the following conclusion. Suppose that $G$ is a first countable compactly generated locally compact abelian group with non-compact connected component of the identity. If $f \in L^{2}(G)$ is such that $Z f$ is continuous, then the functions $\varphi_{k, \gamma}$ do not form a frame for $L^{2}(G)$.

\section{ACKNOWLEDGMENT}

The authors are indebted to the referee for pointing out some misprints and

providing an example of a locally compact abelian group which contains no uniform lattice.

\section{REFERENCES}

[1] L. Auslander and R. Tolimieri, Abelian harmonic analysis, theta functions and function algebras on a nilmanifold, Lecture Notes in Mathematics 436, Springer-Verlag, Berlin/Heidelberg/New York, 1975. MR 54:2877

[2] J. J. Benedetto and M. W. Frazier, Wavelets: Mathematics and applications, CRC Press, Boca Raton, 1994. MR 94f: 42048

[3] M. Boon and J. Zak, Amplitudes on von Neumann lattices, J. Math. Phys. 22 (1981), 10901099. MR 84e:81029

[4] C. E. Heil and D. F. Walnut, Continuous and discrete wavelet transforms, SIAM Review 31 (1989), 628-666. MR 91c:42032

[5] E. Hewitt and K. A. Ross, Abstract harmonic analysis. I, II, Springer-Verlag, Berlin/Heidelberg/New York, 1963. MR 28:158; MR 41:7378

[6] A. J. E. M. Janssen, Bargmann transform, Zak transform, and coherent states, J. Math. Phys. 23 (1982), 720-731. MR 84h:81041

[7] A. J. E. M. Janssen, The Zak transform: A signal transform for sampled time-continuous signals, Philips J. Res. 43 (1988), 23-69. MR 89g:94005 
[8] G. W. Mackey, Induced representations of locally compact groups. I, Ann. of Math. (2) 55 (1952), 101-139. MR 13:434a

[9] G. W. Mackey, Borel structure in groups and their duals, Trans. Amer. Math. Soc. 85 (1957), 134-165. MR 19:752b

[10] D. Montgomery and L. Zippin, Topological transformation groups, Interscience, New York, 1955. MR 17:383b

[11] J. Zak, Finite translations in solid state physics, Phys. Rev. Letters 19 (1967), 1385-1387.

Fachbereich Mathematik/Informatik, Universität Paderborn, 33095 Paderborn, GERMANY

E-mail address: kaniuth@uni-paderborn.de

E-mail address: gittak@uni-paderborn.de 